\title{
First-principles calculations of phase transition, low elastic modulus, and superconductivity for zirconium
}

\author{
Bao-Tian Wang, $1,2, *$ Peng Zhang, ${ }^{3}$ Han-Yu Liu, ${ }^{4}$ Wei-Dong Li, ${ }^{1}$ and Ping Zhang ${ }^{2,5,+\dagger}$ \\ ${ }^{1}$ Institute of Theoretical Physics and Department of Physics, \\ Shanxi University, Taiyuan 030006, People's Republic of China \\ ${ }^{2} L C P$, Institute of Applied Physics and Computational Mathematics, \\ P.O. Box 8009, Beijing 100088, People's Republic of China \\ ${ }^{3}$ Department of Nuclear Science and Technology, \\ Xi'an Jiaotong University, Xi'an 710049, People's Republic of China \\ ${ }^{4}$ State Key Lab of Superhard Materials, Jilin University, \\ Changchun 130012, People's Republic of China \\ ${ }^{5}$ Center for Applied Physics and Technology, \\ Peking University, Beijing 100871, People's Republic of China
}

\begin{abstract}
The elasticity, dynamic properties, and superconductivity of $\alpha, \omega$, and $\beta \mathrm{Zr}$ are investigated by using first-principles methods. Our calculated elastic constants, elastic moduli, and Debye temperatures of $\alpha$ and $\omega$ phases are in excellent agreement with experiments. Electron-phonon coupling constant $\lambda$ and electronic density of states at the Fermi level $N\left(E_{\mathrm{F}}\right)$ are found to increase with pressure for these two hexagonal structures. For cubic $\beta$ phase, the critical pressure for mechanical stability is predicted to be $3.13 \mathrm{GPa}$ and at $P=4 \mathrm{GPa}$ the low elastic modulus $(E=31.97$ GPa) can be obtained. Besides, the critical pressure for dynamic stability of $\beta$ phase is achieved by phonon dispersion calculations to be $\sim 26 \mathrm{GPa}$. Over this pressure, $\lambda$ and $N\left(E_{\mathrm{F}}\right)$ of $\beta$ phase decrease upon further compression. Our calculations show that the large value of superconducting transition temperature $T_{\mathrm{c}}$ at $30 \mathrm{GPa}$ for $\beta \mathrm{Zr}$ is mainly due to the TA1 soft mode. Under further compression, the soft vibrational mode will gradually fade away.
\end{abstract}

PACS numbers: 62.20.Dc, 64.70.kd, 61.50.Ks, 74.70.Ad

*E-mail: wbt11129@sxu.edu.cn

${ }^{\dagger}$ Corresponding author; zhang_ping@iapcm.ac.cn 


\section{INTRODUCTION}

Group IV transition metals such as titanium, zirconium, and hafnium have wide applications in the aerospace, nuclear, and biomedical industry [1-3]. The body-centered cubic (bcc) type ( $\beta$ phase) of $\mathrm{Ti}$ and $\mathrm{Zr}$ alloys in usage as metallic biomaterial have been studied extensively because of their properties of nontoxicity, high strength, good biocompatibility, and low elastic modulus [1, 4]. However, the investigations of the single crystal $\beta \mathrm{Zr}$ as biomaterial has not been reported.

Actually, at ambient condition Zr crystallizes in hexagonal closed-packed (hcp) structure ( $\alpha$ phase). And at high temperature of $1135 \mathrm{~K}$, it transforms into the $\beta$ phase. At room temperature and under pressure range of 2-7 GPa [5-7], the $\alpha$ phase transforms to another more open hexagonal structure of $\omega$ phase with space group P6/mmm (No. 191). And under further high pressure of 30-35 GPa, the $\omega \rightarrow \beta$ phase transition has been observed [6, 8 11]. The Young's moduli of both $\alpha$ and $\omega$ phases at ambient condition are largely bigger than that of a human bone (about $30 \mathrm{GPa}$ ). So the condition of low elastic modulus cannot be satisfied for these two phases. As for $\beta$ phase, it cannot be exploited because the reverse phase transformations take place upon unloading. So it seems that we should not consider metal $\mathrm{Zr}$ as candidate biomaterial. But, fortunately recent experiment has successfully stabilized the $\beta \mathrm{Zr}$ at room temperature using compression stresses higher than $3 \mathrm{GPa}$ plus the controlled application of shear [3]. This makes stabilizing the high temperature or pressure phases of Zr at ambient conditions reality. Therefore, the study of the elastic modulus for $\beta \mathrm{Zr}$ at low pressure is valuable.

As another key objective of our work, we calculate the superconducting transition temperature $T_{c}$ for metal Zr. Although the $T_{c}$ of $\mathrm{Zr}$ has been observed in experiments [9], to date no theoretical work is found in the literature. Our calculations will show that the increase (decrease) in $T_{c}$ with pressure can be understood in terms of the enhancement (reduction) of the electronic density of states (DOS) at the Fermi level and the corresponding behaviors of electron-phonon coupling constant. In present study, the dynamic stability of $\beta \mathrm{Zr}$ is tested carefully by performing phonon dispersion calculations. The $\beta \rightarrow \omega$ and $\beta \rightarrow \alpha$ transitions are discussed in detail. The rest of this paper is arranged as follows. In Sec. II the computational methods are described. In Sec. III we present and discuss our results. In Sec. IV we summarize the conclusions of this work. 


\section{COMPUTATIONAL METHODS}

\section{A. Computational details}

The first-principles density functional theory (DFT) calculations on the basis of the frozen-core projected augmented wave (PAW) method of Blöchl [12] are performed within the Vienna ab initio simulation package (VASP) [13], where the Perdew, Burke, and Ernzerhof (PBE) [14] form of the generalized gradient approximation (GGA) is employed to describe electron exchange and correlation. For the plane-wave set, a cutoff energy of 500 $\mathrm{eV}$ is used. The $\Gamma$-centered $k$ point-meshes in the full wedge of the Brillouin zone (BZ) are sampled by $18 \times 18 \times 16,16 \times 16 \times 18$, and $18 \times 18 \times 18$ grids according to the Monkhorst-Pack (MP) [15] for $\alpha$ (two-atom cell), $\omega$ (three-atom cell), and $\beta$ (two-atom cell) Zr, respectively, and all atoms are fully relaxed until the Hellmann-Feynman forces become less than 0.001 $\mathrm{eV} / \AA$. The $\mathrm{Zr} 4 d^{3} 5 s^{1}$ orbitals are explicitly included as valence electrons. The pseudopotential plane-wave method within the PBE-GGA through the QUANTUM-ESPRESSO package [16] is employed to calculate the electronic properties, lattice dynamics, and electron-phonon coupling (EPC) for $\mathrm{Zr}$, where the $\mathrm{Zr} 4 s^{2} 4 p^{6} 4 d^{2} 5 s^{2}$ are treated as valence electrons. Convergence tests give the choice of kinetic energy cutoffs of 60 Ry with Gaussians width of 0.05 Ry for all three phases of $\mathrm{Zr}$ and $12 \times 12 \times 8,8 \times 8 \times 12$, and $16 \times 16 \times 16 \mathrm{MP}$ grids of $k$-point meshes for $\alpha, \omega$, and $\beta \mathrm{Zr}$, respectively. Phonon frequencies are calculated based on the density functional linear-response method [17, 18]. In the interpolation of the force constants for the phonon dispersion curve calculations, $6 \times 6 \times 4,4 \times 4 \times 6$, and $8 \times 8 \times 8 q$-point meshes and denser $24 \times 24 \times 16,16 \times 16 \times 24$, and $32 \times 32 \times 32 k$-point meshes in the first BZ are used for $\alpha, \omega$, and $\beta$ phases of $\mathrm{Zr}$, respectively.

\section{B. Mechanical properties}

To avoid the Pulay stress problem, the geometry optimization at each volume is performed at fixed volume rather than constant pressure. The theoretical equilibrium volume, bulk modulus $B$, and pressure derivative of the bulk modulus $B^{\prime}$ are obtained by fitting the energy-volume data in the third-order Birch-Murnaghan equation of state (EOS) [19]. Elastic constants for cubic symmetry $\left(C_{11}, C_{12}\right.$, and $\left.C_{44}\right)$ and hexagonal structure $\left(C_{11}\right.$, $C_{12}, C_{13}, C_{33}$, and $\left.C_{44}\right)$ are calculated by applying stress tensors with various small strains 
onto the equilibrium structures. The strain amplitude $\delta$ is varied in steps of 0.006 from $\delta=-0.036$ to 0.036 . After obtaining elastic constants, the polycrystalline bulk modulus $B$ and shear modulus $G$ are calculated from the Voigt-Reuss-Hill (VRH) approximations [20]. The Young's modulus $E$ and Poisson's ratio $v$ are calculated through $E=9 B G /(3 B+G)$ and $v=(3 B-2 G) /[2(3 B+G)]$. In calculation of the Debye temperature $\left(\theta_{D}\right)$, we use the relation

$$
\theta_{D}=\frac{h}{k_{B}}\left(\frac{3 n}{4 \pi \Omega}\right)^{1 / 3} v_{m}
$$

where $h$ and $k_{B}$ are Planck and Boltzmann constants, respectively, $n$ is the number of atoms in the molecule, $\Omega$ is molecular volume, and $v_{m}$ is the average sound wave velocity. The average wave velocity in the polycrystalline materials is approximately given as

$$
v_{m}=\left[\frac{1}{3}\left(\frac{2}{v_{t}^{3}}+\frac{1}{v_{l}^{3}}\right)\right]^{-1 / 3},
$$

where $v_{t}=\sqrt{G / \rho}\left(\rho\right.$ is the density) and $v_{l}=\sqrt{(3 B+4 G) / 3 \rho}$ are the transverse and longitudinal elastic wave velocity of the polycrystalline materials, respectively.

\section{Superconduntivity}

The superconducting transition temperature $T_{c}$ is evaluated by using the Allen-Dynes modified McMillan equation [21, 22]

$$
T_{c}=\frac{\omega_{\log }}{1.2} \exp \left[-\frac{1.04(1+\lambda)}{\lambda-\mu^{*}(1+0.62 \lambda)}\right],
$$

where

$$
\omega_{\log }=\exp \left[\frac{2}{\lambda} \int_{0}^{\infty} \frac{d \omega}{\omega} \alpha^{2} F(\omega) \ln \omega\right]
$$

is the logarithmic average frequency,

$$
\lambda=2 \int_{0}^{\infty} \frac{\alpha^{2} F(\omega)}{\omega} d \omega
$$

is the electron-phonon coupling constant, and $\mu^{*}$ is the Coulomb pseudopotential. The Eliashberg electron-phonon spectral function $\alpha^{2} F(\omega)$ is written as

$$
\alpha^{2} F(\omega)=\frac{1}{2 \pi N\left(E_{\mathrm{F}}\right)} \sum_{q v} \frac{\gamma_{q v}}{\omega_{q v}} \delta\left(\omega-\omega_{q v}\right),
$$

where $N\left(E_{\mathrm{F}}\right)$ is the electronic density of state (DOS) at the Fermi level. 


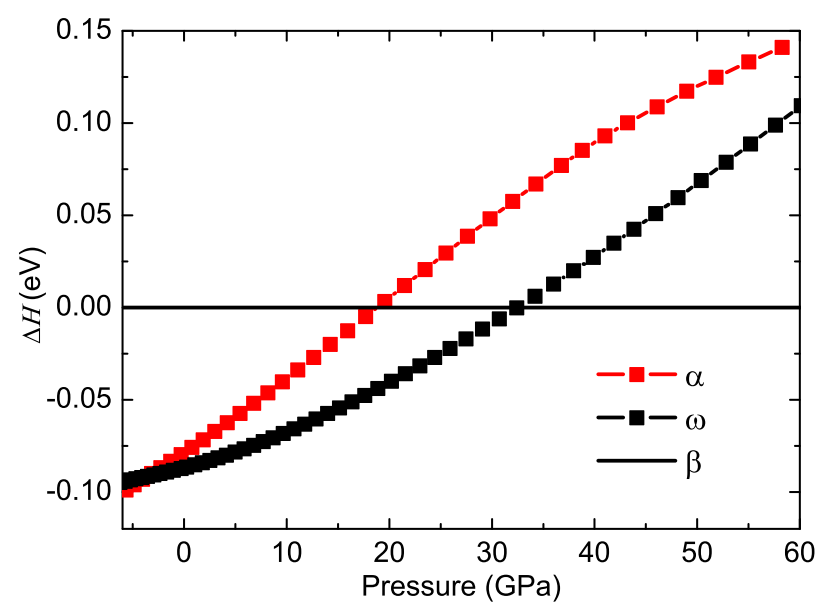

FIG. 1: (Color online) Calculated enthalpy differences of $\alpha$ and $\omega$ phases with respect to $\beta$ phase as functions of pressure.

\section{RESULTS}

At $0 \mathrm{~K}$, the Gibbs free energy is equal to the enthalpy $H$. After calculation, we can plot in Fig. 1 the relative enthalpies of the $\alpha$ and the $\omega$ phases with respect to the $\beta$ phase as functions of pressure. The crossing between the $\alpha$ and $\omega$ enthalpy curves readily gives phase transition pressure of $-3.7 \mathrm{GPa}$, which indicates that at ambient pressure the $\omega$ phase is more stable than the $\alpha$ phase. This fact is in disagreement with experiment, but coincides well with recent DFT-PBE [23] and FP-LAPW [24] calculations. The disagreement between theory and experiment mainly originates from temperature effect. Actually, previous DFTPBE [23] study clearly shows that the transition of $\alpha \rightarrow \omega$ occurs at $1.7 \mathrm{GPa}$ at $T=300 \mathrm{~K}$. As for the $\omega \rightarrow \beta$ transition, a pressure of $32.4 \mathrm{GPa}$ is obtained. This value falls exactly in the pressure range of 30-35 GPa measured by experiments [6, $8-11]$ and also fairly accords with other calculations [23, 24].

In Table \, we report our calculated lattice parameters, bulk modulus, pressure derivative of the bulk modulus, and elastic constants of $\alpha, \omega$, and $\beta \mathrm{Zr}$ at different pressures. For comparison, the experimental values [2, 7, 25 27] and other theoretical results [1, 28, 29] are also listed. Obviously, our calculated results accord well with experiments and other calculations for $\alpha$ and $\omega$ phases. However, for $\beta$ Zr the difference of lattice parameter and bulk modulus between our calculation and the experiment from Zhao et al. [7] is evident. This is due to the fact that the experiment is conducted at $T=973 \mathrm{~K}$, while our calculation 
TABLE I: Calculated lattice parameters ( $a$ or $c$ ), bulk modulus $(B)$, pressure derivative of the bulk modulus $\left(B^{\prime}\right)$, and elastic constants of $\alpha, \omega$, and $\beta \mathrm{Zr}$ at different pressures. For comparison, experimental values and other theoretical results are also listed.

\begin{tabular}{|c|c|c|c|c|c|c|c|c|c|c|c|}
\hline Phase & Method & $\begin{array}{c}\text { Pressure } \\
(\mathrm{GPa})\end{array}$ & $\begin{array}{c}a \\
(\AA)\end{array}$ & $\begin{array}{c}\mathrm{c} \\
(\AA)\end{array}$ & $\begin{array}{c}B \\
(\mathrm{GPa})\end{array}$ & $B^{\prime}$ & $\begin{array}{c}C_{11} \\
(\mathrm{GPa})\end{array}$ & $\begin{array}{c}C_{12} \\
(\mathrm{GPa})\end{array}$ & $\begin{array}{c}C_{13} \\
(\mathrm{GPa})\end{array}$ & $\begin{array}{c}C_{33} \\
(\mathrm{GPa})\end{array}$ & $\begin{array}{c}C_{44} \\
(\mathrm{GPa})\end{array}$ \\
\hline \multirow[t]{5}{*}{$\alpha$} & This work & 0 & 3.236 & 5.168 & 96.0 & 3.12 & 146.7 & 68.5 & 71.0 & 163.3 & 26.0 \\
\hline & & 5.35 & 3.178 & 5.093 & & & 162.3 & 78.4 & 81.9 & 187.5 & 24.9 \\
\hline & DFT-PBE $^{a}$ & 0 & 3.240 & 5.178 & 93.4 & 3.22 & 141.1 & 67.6 & 64.3 & 166.9 & 25.8 \\
\hline & $\mathrm{DFT}_{-\mathrm{PBE}^{b}}$ & 0 & 3.232 & 5.182 & 93.4 & 3.22 & 139.4 & 71.3 & 66.3 & 162.7 & 25.5 \\
\hline & Expt. & 0 & $3.233^{c}$ & $5.146^{c}$ & $92^{c}$ & $4.0^{c}$ & $144.0^{d}$ & $74.0^{d}$ & $67.0^{d}$ & $166.0^{d}$ & $33.0^{d}$ \\
\hline \multirow[t]{5}{*}{$\omega$} & This work & 0 & 5.036 & 3.152 & 96.9 & 3.39 & 161.7 & 72.6 & 53.5 & 195.6 & 33.7 \\
\hline & & 6.04 & 4.939 & 3.097 & & & 187.0 & 85.8 & 63.1 & 224.4 & 37.3 \\
\hline & & 10.01 & 4.884 & 3.066 & & & 201.5 & 94.5 & 69.0 & 241.1 & 38.8 \\
\hline & DFT-PBE $^{a}$ & 0 & 5.056 & 3.150 & 101.1 & 3.27 & 165.2 & 75.6 & 47.5 & 198.7 & 30.6 \\
\hline & Expt. & 0 & $5.039^{e}$ & $3.150^{e}$ & $104.0^{f}$ & $2.8^{f}$ & & & & & \\
\hline \multirow[t]{11}{*}{$\beta$} & This work & 0 & 3.574 & & 90.2 & 3.06 & 86.6 & 92.3 & & & 26.6 \\
\hline & & 10 & 3.461 & & & & 123.1 & 106.6 & & & 32.0 \\
\hline & & 20 & 3.374 & & & & 160.0 & 116.8 & & & 37.0 \\
\hline & & 30 & 3.302 & & & & 196.9 & 124.1 & & & 42.2 \\
\hline & & 35 & 3.271 & & & & 216.7 & 124.4 & & & 45.8 \\
\hline & & 40 & 3.241 & & & & 235.2 & 128.4 & & & 49.2 \\
\hline & & 50 & 3.188 & & & & 265.6 & 136.2 & & & 54.4 \\
\hline & & 60 & 3.141 & & & & 298.3 & 141.3 & & & 61.7 \\
\hline & $\mathrm{DFT}_{-\mathrm{PBE}}{ }^{b}$ & 0 & 3.580 & & & & 84.2 & 91.4 & & & 32.3 \\
\hline & $\mathrm{LDA}^{g}$ & 0 & & & & & & & & & 32.8 \\
\hline & Expt. & 0 & 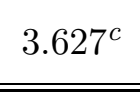 & & $\square \quad 6^{66^{c}}$ & & $104^{h}$ & $93^{h}$ & $\square$ & & $\begin{array}{c}38^{h} \\
\end{array}$ \\
\hline
\end{tabular}




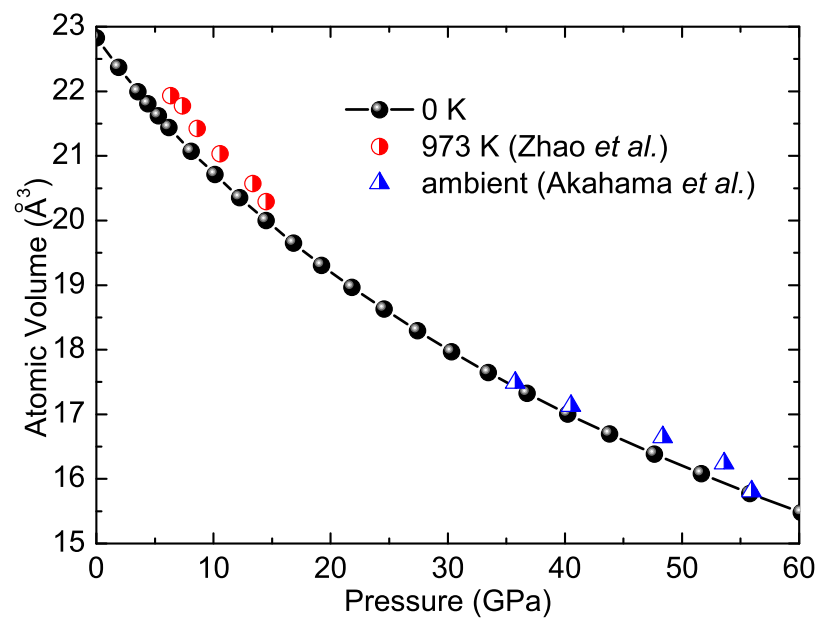

FIG. 2: (Color online) Calculated compression curves of $\beta \mathrm{Zr}$ compared with experimental measurements.

is valid only at $0 \mathrm{~K}$. Similar temperature effect for $\beta \mathrm{Zr}$ is also found in studying the compression behaviour. As shown in Fig. 2, although good consistence with the experiment [10] at high pressure domain is clear, the values of the atomic volumes are smaller than that by experiment at $T=973 \mathrm{~K}$. Nevertheless, the compression performance of $\beta \mathrm{Zr}$ is wholly consistent with experiments. From Table 【, one can find that the difference of elastic constants at zero pressure between calculation and experiment is evident. This also can be attributed to the temperature effect. In fact, the good accordance of the lattice parameter and elastic constants of $\beta \mathrm{Zr}$ at $0 \mathrm{GPa}$ between our calculation and previous DFT-PBE [1] study supplies the safeguard for our study of structure, mechanical, and electronic properties of $\beta$ Zr. Additionally, the mechanical stability of $\alpha$ and $\omega$ Zr at 0 GPa and at some typical finite pressures can be predicted from the elastic constants data. But the elastic constants of $\beta \mathrm{Zr}$ at $0 \mathrm{GPa}$ illustrate that the cubic phase is mechanically unstable, which is in good agreement with the results by Ahuja et al. [29] and Ikehata et al. [1]. Actually, the mechanical stability of $\beta \mathrm{Zr}$ can be obtained through applying external pressure and our data show that along with the increase of pressure from $0 \mathrm{GPa}$ to $60 \mathrm{GPa}$, the value of $C_{11}-C_{12}$ increases almost linearly from $-5.7 \mathrm{GPa}$ to $157 \mathrm{GPa}$. Fitting the curve (not shown) of the pressure behaviour of $C_{11}-C_{12}$ by first-order polynomial function, we find that the value of $C_{11}-C_{12}$ becomes positive at $P \geq 3.13 \mathrm{GPa}$. In fact, at $P=4 \mathrm{GPa}$ our first-principles calculated $C_{11}, C_{12}$, and $C_{44}$ equal to $101.8,98.2$, and $28.7 \mathrm{GPa}$, respectively, 
TABLE II: Calculated elastic moduli, Poisson's ratio $(v)$, density $(\rho)$, transverse $\left(v_{t}\right)$, longitudinal $\left(v_{l}\right)$, and average $\left(v_{m}\right)$ sound velocities calculated from polycrystalline bulk and shear modulus, and Debye temperature of $\alpha, \omega$, and $\beta \mathrm{Zr}$ at different pressures. For comparison, experimental values and other theoretical results are also listed.

\begin{tabular}{|c|c|c|c|c|c|c|c|c|c|c|c|}
\hline Phase & Method & $\begin{array}{c}\text { Pressure } \\
(\mathrm{GPa})\end{array}$ & $\begin{array}{c}B \\
(\mathrm{GPa})\end{array}$ & $\begin{array}{c}G \\
(\mathrm{GPa})\end{array}$ & $\begin{array}{c}E \\
(\mathrm{GPa})\end{array}$ & $v$ & $\begin{array}{c}\rho \\
\left(\mathrm{g} / \mathrm{cm}^{3}\right)\end{array}$ & $\begin{array}{c}v_{t} \\
(\mathrm{~km} / \mathrm{s})\end{array}$ & $\begin{array}{c}v_{l} \\
(\mathrm{~km} / \mathrm{s})\end{array}$ & $\begin{array}{c}v_{m} \\
(\mathrm{~km} / \mathrm{s})\end{array}$ & $\begin{array}{l}\theta_{D} \\
(\mathrm{~K})\end{array}$ \\
\hline \multirow[t]{3}{*}{$\alpha$} & This work & 0 & 97.4 & 33.8 & 91.0 & 0.344 & 6.465 & 2.288 & 4.695 & 2.571 & 267.3 \\
\hline & & 5.35 & 110.4 & 34.9 & 94.8 & 0.357 & 6.804 & 2.266 & 4.803 & 2.550 & 269.8 \\
\hline & Expt. ${ }^{a}$ & 0 & 95.3 & 36.3 & 96.6 & 0.331 & 6.511 & 2.361 & 4.698 & & 275.9 \\
\hline \multirow[t]{6}{*}{$\omega$} & This work & 0 & 97.5 & 43.6 & 113.7 & 0.306 & 6.568 & 2.575 & 4.868 & 2.878 & 300.9 \\
\hline & & 6.04 & 113.5 & 49.0 & 128.5 & 0.311 & 6.948 & 2.655 & 5.074 & 2.970 & 276.4 \\
\hline & & 10.01 & 123.2 & 51.6 & 135.8 & 0.316 & 7.177 & 2.681 & 5.172 & 3.001 & 282.3 \\
\hline & Expt. $^{a}$ & 0 & 104.0 & 45.1 & 118.3 & 0.311 & 6.589 & 2.616 & 4.996 & & 306.1 \\
\hline & & 6.88 & & & & & & 2.638 & 5.177 & & \\
\hline & & 10.59 & & & & & & 2.652 & 5.284 & & \\
\hline \multirow[t]{8}{*}{$\beta$} & This work & 10 & 112.1 & 18.7 & 53.1 & 0.421 & 7.309 & 1.599 & 4.330 & 1.816 & 196.7 \\
\hline & & 20 & 131.2 & 29.8 & 83.2 & 0.394 & 7.889 & 1.944 & 4.655 & 2.199 & 244.4 \\
\hline & & 30 & 148.4 & 39.8 & 109.6 & 0.377 & 8.415 & 2.175 & 4.893 & 2.454 & 278.7 \\
\hline & & 35 & 156.5 & 45.5 & 124.6 & 0.367 & 8.662 & 2.293 & 5.008 & 2.584 & 296.3 \\
\hline & & 40 & 164.0 & 50.9 & 138.3 & 0.360 & 8.900 & 2.390 & 5.104 & 2.691 & 311.4 \\
\hline & & 50 & 179.3 & 58.3 & 157.8 & 0.353 & 9.353 & 2.497 & 5.243 & 2.809 & 330.4 \\
\hline & & 60 & 193.6 & 68.0 & 182.5 & 0.343 & 9.781 & 2.636 & 5.391 & 2.961 & 353.5 \\
\hline & Expt. $^{b}$ & 0 & 96.7 & 18.1 & 51.2 & 0.412 & 6.420 & 1.681 & 4.339 & 1.906 & $197.8,177^{c}$ \\
\hline
\end{tabular}

${ }^{a}$ Reference [30], ${ }^{b}$ Calculated using present scheme with elastic constants at $T=915^{\circ} \mathrm{C}$ from reference [26], ${ }^{c}$ Reference [26].

which explicitly indicate the elastic stability of bcc Zr under this pressure.

After obtaining elastic constants, the elastic moduli, Poisson's ratio $(v)$, transverse sound velocities $\left(v_{t}\right)$, longitudinal sound velocities $\left(v_{l}\right)$, average sound velocities $\left(v_{m}\right)$, and Debye temperature of $\alpha, \omega$, and $\beta$ Zr at different pressures are calculated and tabulated in Table II. The experimental values [26, 30] are also collected in Table II for comparison. The excellent 

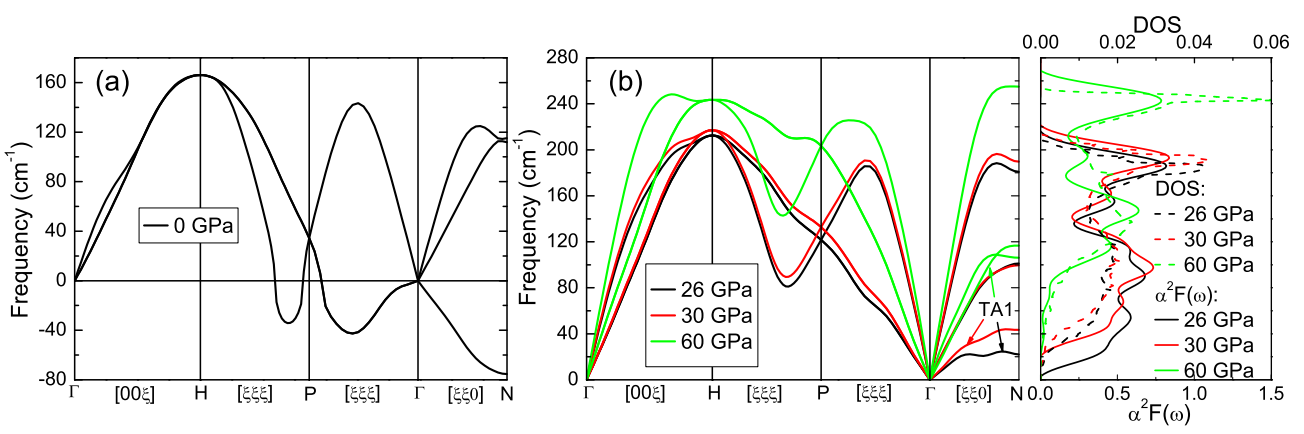

FIG. 3: (Color online) Phonon dispersion at 0 GPa (a) and phonon dispersion, Eliashberg phonon spectral function, and phonon DOS at 26, 30, and 60 GPa (b) for $\beta$ Zr.

coincidence between our calculation and experiment for $\alpha$ and $\omega$ phases can be seen. For $\beta$ phase, the bulk modulus $B$, shear modulus $G$, Young's modulus $E$, and Debye temperature all linearly increase with pressure. The Young's modulus $E$ at $P=4 \mathrm{GPa}$ (just over the critical pressure $3.13 \mathrm{GPa}$ for the elastic stability of $\beta \mathrm{Zr}$ ) is calculated to equal to 31.97 GPa, which is very near the Young's modulus value ( $30 \mathrm{GPa}$ ) of a human bone. Thus, we successfully indicate the importance of stabilizing the high temperature or pressure phase of $\mathrm{Zr}$ at ambient conditions. In Table III, we also list the results of $\beta$ phase calculated using present scheme with elastic and lattice constants at $T=915^{\circ} \mathrm{C}$ from Ref. [26]. The derived Debye temperature is slightly higher than that given in Ref. [26].

Phonon dispersion results for $\beta \mathrm{Zr}$ at selected pressures are shown in Fig. 3, Like the $\beta$ phase of Ti and $\mathrm{Hf}[31,32]$, the high pressure $\beta$ phase of $\mathrm{Zr}$ is also dynamically unstable at low pressure [Fig. 3(a)]. Our phonon dispersion calculations at high pressure illustrate that the $\beta \mathrm{Zr}$ becomes dynamically stable at around $26 \mathrm{GPa}$. In fact, no imaginary frequencies are observed at 26-60 GPa pressure domain as clearly indicated in Fig. 3(b). Therefore, although recent experiment by Pérez-Prado and Zhilyaev [3] has successfully stabilized the high pressure/temperature phase of $\mathrm{Zr}$ at ambient condition, our phonon dispersion calculations illustrate that the $\beta$ phase is dynamically unstable in pressure domain of $0-25 \mathrm{GPa}$ at $0 \mathrm{~K}$. This fact confirms that the temperature effect on the phase stability is also critical for the experimentally observed [3] $\beta$ phase at ambient condition. Besides, the dynamical stability test at 26-60 GPa supplies the safeguard for our following study of the superconductivity of $\beta \mathrm{Zr}$ at high pressure. As shown in Fig. 3(a), our phonon dispersion results indicate soft modes for the longitudinal $\mathrm{L} \frac{2}{3}[111]$ and transverse $\mathrm{T} \frac{1}{2}[110]$ phonons. 


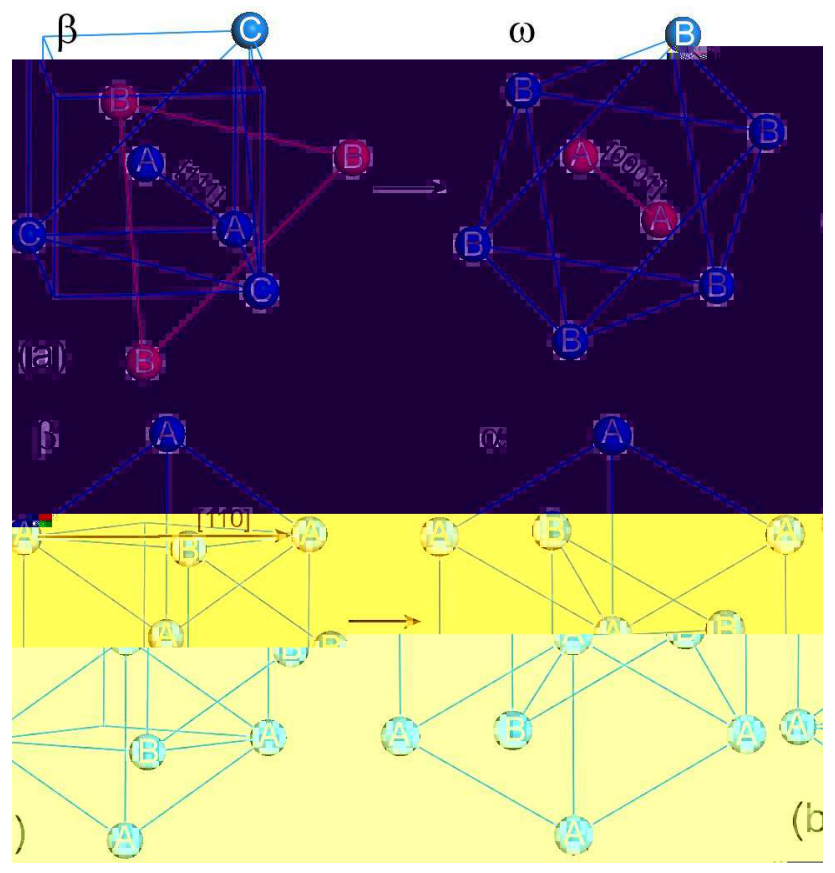

FIG. 4: (Color online) Schematic illustrations of the structural transition for (a) $\beta \rightarrow \omega$ and (b) $\beta \rightarrow \alpha$.

As indicated by experiment [26], the L $\frac{2}{3}[111]$ unstable phonon branch is responsible for the $\beta \rightarrow \omega$ transition and the $\mathrm{T} \frac{1}{2}[110]$ implies the $\beta \rightarrow \alpha$ transition. In Fig. 4, we show the paths of $\beta \rightarrow \omega$ and $\beta \rightarrow \alpha$ transitions. As shown in Fig. 4(a), the bcc $\beta$ phase can be viewed as ABC periodically layered structure along the [111] direction and there are two periods of $\mathrm{ABC}$ layers in its unit cell. For $\omega$ phase, it can be viewed as $\mathrm{AB}$ periodical layered structure along the [0001] direction [Fig. 4(a)]. The atoms on B and C layers in $\beta$ phase move easily along the soft mode direction of [111] and can transfer to be the B layer of $\omega$ phase. Therefore, the atomic displacement along the [111] direction is the main origin for the $\beta \rightarrow \omega$ phase transition. For $\beta \rightarrow \alpha$ transition, one can regard the $\beta$ phase as two layered (AB) structure along the $[1 \overline{1} 0]$ orientation [Fig. 4(b)]. In transition, firstly the atoms on each layer expand along the [110] direction to form a hexagon, and second, the adjacent (110) planes slip relatively along the [110] direction to create the hcp structure.

For high pressure phase of Zr, it has evident predominance for high-temperature superconductivity. The EPC calculations have been performed to explore the superconductivity. The Eliashberg phonon spectral function $\alpha^{2} F(\omega)$ and phonon DOS at 26, 30, and 60 GPa for $\beta \mathrm{Zr}$ are shown in Fig. 3(b). Logarithmic average phonon frequency $\omega_{\log }$ and the EPC 
TABLE III: Calculated logarithmic average of vibrational frequencies $\omega_{\log }$ and EPC constant $\lambda$ of $\alpha, \omega$, and $\beta \mathrm{Zr}$ at different pressures.

\begin{tabular}{cccc}
\hline \hline Phase & $\begin{array}{c}\text { Pressure } \\
(\mathrm{GPa})\end{array}$ & $\omega_{\log }$ & $\lambda$ \\
\hline$\alpha$ & 0 & $(\mathrm{~K})$ & \\
\hline & 5 & 142.52 & 0.8611 \\
$\omega$ & 0 & 139.26 & 0.8852 \\
& 10 & 168.29 & 0.6723 \\
$\beta$ & 20 & 182.27 & 0.6753 \\
& 30 & 186.51 & 0.7302 \\
& 26 & 184.06 & 0.8174 \\
& 28 & 80.91 & 2.5311 \\
& 30 & 123.43 & 2.0237 \\
& 40 & 136.22 & 1.7822 \\
& 50 & 169.17 & 1.2745 \\
& 60 & 198.83 & 1.0147 \\
\hline \hline
\end{tabular}

strength $\lambda$ of $\alpha, \omega$, and $\beta$ Zr at different pressures are listed in Table III. The superconducting $T_{\mathrm{c}}$ has been estimated from Eqs. (3), and a typical value of Coulomb pseudopotential $\mu^{*}=0.12$ is used. Our calculated $T_{\mathrm{c}}$, together with $N\left(E_{\mathrm{F}}\right)$, as a function of pressure for the three phases are plotted in Fig. 5. Note that there are two different kinds of atoms for $\omega \mathrm{Zr}$ : Zr1 in $1 a$ and Zr2 in $2 d$ sites. Clearly, the predicted $T_{\mathrm{c}}$ is in good agreement with experimental results. As indicated by McMilan equation [Eqs. (3)], $T_{\mathrm{c}}$ has tight relationship with $\omega_{\log }, \lambda$, and $N\left(E_{\mathrm{F}}\right)$. From 0-7 GPa, total $N\left(E_{\mathrm{F}}\right)$ of $\alpha$ phase increases with pressure. In pressure range of 7-30 GPa, the average $N\left(E_{\mathrm{F}}\right)$ of $\omega$ phase over two kinds of atomic arrangements also exhibits increasing behavior upon compression. In contrast, total $N\left(E_{\mathrm{F}}\right)$ of $\beta \mathrm{Zr}$ decreases with pressure in the whole domain of 26-60 GPa. All these pressure-dependent behaviors of $N\left(E_{\mathrm{F}}\right)$ obey one known fact that a more stable phase has a lower value of $N\left(E_{\mathrm{F}}\right)$. Through plotting partial $N\left(E_{\mathrm{F}}\right)$ of $s, p$, and $d$ orbitals (not shown), we find that the main contribution to the pressure-dependent behavior of the total $N\left(E_{\mathrm{F}}\right)$ comes from $d$ orbital. The $s$ orbital has no contribution and the contribution from $p$ orbital is limited. 


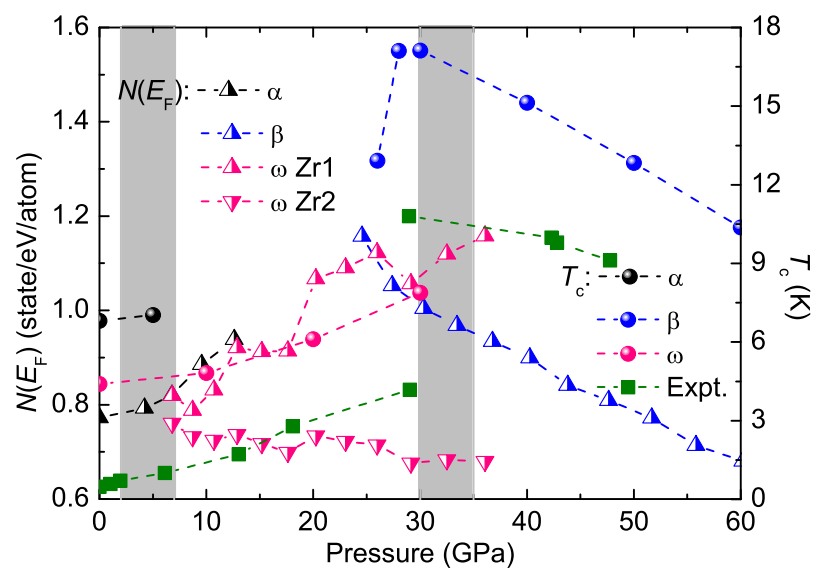

FIG. 5: (Color online) Calculated total density of state at the Fermi level $N\left(E_{\mathrm{F}}\right)$ and superconducting transition temperature $T_{c}$ as functions of pressure for $\alpha, \omega$, and $\beta \mathrm{Zr}$. The experimental values of $T_{c}$ from Ref. [9] are also presented. The two shaded regions indicate the $\alpha \rightarrow \omega$ and $\omega \rightarrow \beta$ phase transition ranges of 2-7 GPa [5-7] and 30-35 GPa [6, 8-11], respectively.

From Fig. 5, one can see that the experimental $T_{\mathrm{c}}$ changes in almost the same way as that of the $N\left(E_{\mathrm{F}}\right)$. So, it seems that relative larger value of the $N\left(E_{\mathrm{F}}\right)$ for $\beta$ Zr at 26 GPa may predict larger $T_{\mathrm{c}}$ for superconductivity. However, $N\left(E_{\mathrm{F}}\right)$ is less directly important than $\omega_{\log }$ and $\lambda$ for $T_{\mathrm{c}}$.

From Table III, one can see that the calculated $\lambda$ for $\alpha$ and $\omega$ phases increases with pressure while $\omega_{\log }$ does not show any clear dependence on pressure. As a result, the increasing behavior of $T_{\mathrm{c}}$ upon compression is mainly due to the increase of $\lambda$. For $\beta \mathrm{Zr}, \omega_{\log }$ and $\lambda$ have an almost opposite evolution with pressure. However, the changing rate for $\omega_{\log }$ and $\lambda$ is different. The increasing rate of $\omega_{\log }$ is clearly larger than the decreasing rate of $\lambda$ in pressure range of 26 to $30 \mathrm{GPa}$. After being compressed over $30 \mathrm{GPa}$, the decreasing rate of $\lambda$ exceeds the increasing rate of $\omega_{\log }$. So the main affection to the pressure performance of $T_{\mathrm{c}}$ comes from the increase of $\omega_{\log }$ in pressure domain 26-30 GPa, while in 30-60 GPa is from the decrease of $\lambda$. Therefore, the relatively small value of $T_{\mathrm{c}}$ at $26 \mathrm{GPa}$ for $\beta \mathrm{Zr}$ is understandable. Furthermore, the lowest transverse-acoustics (TA1) soft mode for bcc $\beta$ $\mathrm{Zr}$ in pressure range of 26-30 GPa is evident [Fig. 3(b)] and can lead a significant electronphonon contribution. Therefore, as indicated in Table III, the EPC constant $\lambda$ at 26-30 GPa is predominantly larger than that of both $\alpha$ and $\omega$ phases. And, along with increasing the pressure, soft vibrational mode for $\beta$ Zr gradually fade away, resulting in the decrease of $\lambda$ 
and $T_{\mathrm{c}}$. Thus, the decrease of $\lambda$ and $T_{\mathrm{c}}$ for $\beta \mathrm{Zr}$ under pressure mainly originates from the fading of soft vibrational modes induced by increasing pressure.

\section{CONCLUSION}

In summary, the elasticity, dynamic properties, and superconductivity of Zr under pressure up to $60 \mathrm{GPa}$ have been studied by means of the first-principles DFT-PBE method. Our results have shown that the structural parameters, elastic constants, elastic moduli, Poisson's ratio, ultrasonic velocities, and Debye temperature of $\alpha$ and $\omega$ phases coincide well with experiments. The $\alpha \rightarrow \omega$ and $\omega \rightarrow \beta$ phase transition pressures at $T=0 \mathrm{~K}$ have been calculated to be $-3.7 \mathrm{GPa}$ and $32.4 \mathrm{GPa}$, respectively. The compression curve of $\beta \mathrm{Zr}$ is well consistent with experiments. Our calculations have explicitly indicated that the low elastic modulus of $\mathrm{Zr}$ occurs at low pressure in $\beta$ phase.

In phonon dispersion study, the soft modes of $\beta$ phase along [111] and [110] directions have been shown and the $\beta \rightarrow \omega$ and $\beta \rightarrow \alpha$ transition paths have been predicted. Dynamic stability test has illustrated that $\beta \mathrm{Zr}$ is unstable till being compressed over $25 \mathrm{GPa}$. In addition, superconductivity has been obtained by electron-phonon coupling calculations and our calculated $T_{\mathrm{c}}$ accords well with experiments. Through analyzing the electronic density of states at the Fermi level, we have derived that the main contribution to the pressuredependent behavior of the superconductivity comes from the $d$ orbital. The large $T_{\mathrm{c}}$ at around $30 \mathrm{GPa}$ for $\beta \mathrm{Zr}$ is mainly due to the TA1 soft mode. Under pressure, the increase or decrease of $T_{\mathrm{c}}$ for $\mathrm{Zr}$ in all three phases has tight relation with the corresponding behavior of the EPC constant $\lambda$.

\section{Acknowledgments}

This work was partically supported by NSFC under Grants No. 90921003 and No. 60776063, and by the Foundation for Development of Science and Technology of China 
Academy of Engineering Physics under Grant No. 2008B0101008 and No. 2009A0102005.

[1] H. Ikehata, N. Nagasako, T. Furuta, A. Fukumoto, K. Miwa, and T. Saito, Phys. Rev. B 70, $174113(2004)$

[2] W. Liu, B. Li, L. Wang, J. Zhang, and Y. Zhao, Phys. Rev. B 76, 144107 (2007).

[3] M. T. Pérez-Prado and A. P. Zhilyaev, Phys. Rev. Lett. 102, 175504 (2009).

[4] Q. M. Hu, S. J. Li, Y. L. Hao, R. Yang, B. Johansson, and L. Vitos, Appl. Phys. Lett. 93, $121902(2008)$.

[5] S. K. Sikka, Y. K. Vohra, and R. Chidambaram, Prog. Mater. Sci. 27, 245 (1982).

[6] H. Xia, S. J. Duclos, A. L. Ruoff, and Y. K. Vohra, Phys. Rev. Lett. 64, 204 (1990).

[7] Y. S. Zhao, J. Z. Zhang, C. Pantea, J. Qian, L. L. Daemen, P. A. Rigg, R. S. Hixson, G. T. Gray III, Y. P. Yang, L. P. Wang, Y. B. Wang, and T. Y. Uchida, Phys. Rev. B 71, 184119 (2005).

[8] H. Xia, A. L. Ruoff, and Y. K. Vohra, Phys. Rev. B 44, 10374 (1991).

[9] Y. Akahama, M. Kobayashi, and H. Kawamura, J. Phys. Soc. Jpn. 59, 3843 (1990).

[10] Y. Akahama, M. Kobayashi, and H. Kawamura, J. Phys. Soc. Jpn. 60, 3211 (1991).

[11] Y. S. Zhao and J. Z. Zhang, Appl. Phys. Lett. 91, 201907 (2007).

[12] P. E. Blöchl, Phys. Rev. B 50, 17953 (1994).

[13] G. Kresse and J. Furthmüller, Phys. Rev. B 54, 11169 (1996).

[14] J. P. Perdew, K. Burke, and M. Ernzerhof, Phys. Rev. Lett. 77, 3865 (1996).

[15] H. J. Monkhorst and J. D. Pack, Phys. Rev. B 13, 5188 (1972).

[16] A. D. C. S. Baroni, S. de Gironcoli, P. Giannozzi, C. Cavazzoni, G. Ballabio, S. Scandolo, G. Chiarotti, P. Focher, A. Pasquarello, K. Laasonen, A. Trave, R. Car, N. Marzari, and A. Kokalj, http://www.pwscf.org,

[17] S. Baroni, P. Giannozzi, and A. Testa, Phys. Rev. Lett. 58, 1861 (1987).

[18] P. Giannozzi, S. de Gironcoli, P. Pavone, and S. Baroni, Phys. Rev. B 43, 7231 (1991).

[19] F. Birch, Phys. Rev. 71, 809 (1947).

[20] R. Hill, Phys. Phys. Soc. London 65, 349 (1952).

[21] P. B. Allen and R. C. Dynes, Phys. Rev. B 12, 905 (1975).

[22] W. L. McMillan, Phys. Rev. 167, 331 (1968). 
[23] Y. J. Hao, L. Zhang, X. R. Chen, L. C. Cai, Q. Wu, and D. Alfè, Phys. Rev. B 78, 134101 (2008).

[24] I. Schnell and R. C. Albers, J. Phys.: Condens. Matter 18, 1483 (2006).

[25] C. S. Barrett and T. B. Massalski, Structure of Metals (New York: McGraw-Hill, 1966).

[26] A. Heiming, W. Petry, J. Trampenau, M. Alba, C. Herzig, H. R. Schober, and G. Vogl, Phys. Rev. B 43, 10948 (1991).

[27] E. A. Brandes, Smithells Metals Reference Book (Butterworth, London, 1983).

[28] Y. J. Hao, L. Zhang, X. R. Chen, Y. H. Li, and H. L. He, J. Phys.: Condens. Matter 20, 235230 (2008).

[29] R. Ahuja, J. M. Wills, B. Johansson, and O. Eriksson, Phys. Rev. B 48, 16269 (1993).

[30] W. Liu, B. Li, L. Wang, J. Zhang, and Y. Zhao, J. Appl. Phys. 104, 076102 (2008).

[31] P. Souvatzis, O. Eriksson, M. I. Katsnelson, and S. P. Rudin, Phys. Rev. Lett. 100, 095901 (2008).

[32] Z. G. Mei, S. L. Shang, Y. Wang, and Z. K. Liu, Phys. Rev. B 80, 104116 (2009). 